\title{
Origin of the Universe, Dark Energy, and Dark Matter
}

\author{
T. R. Mongan \\ 84 Marin Avenue, Sausalito, California, USA \\ Email: tmongan@gmail.com
}

How to cite this paper: Mongan, T.R. (2018) Origin of the Universe, Dark Energy, and Dark Matter. Journal of Modern Physics, 9, 832-850.

https://doi.org/10.4236/jmp.2018.95054

Received: March 4, 2018

Accepted: April 7, 2018

Published: April 10, 2018

Copyright $\odot 2018$ by author and Scientific Research Publishing Inc. This work is licensed under the Creative Commons Attribution International License (CC BY 4.0).

http://creativecommons.org/licenses/by/4.0/

\begin{abstract}
This explanation of the origin of the universe, dark energy, and dark matter is based on quantum mechanics, general relativity, thermodynamics, and Shannon information theory. It envisions our universe beginning with a quantum fluctuation from nothing into an unstable closed space with our familiar three space dimensions accompanied by seven extra space dimensions, the four basic forces unified, and initial radius of the order of the proton wavelength. The compact extra dimensions collapsed to their ground state with radius of the order of today's Planck length, injecting most of the entropy (information) in the extra dimensions into our three space dimensions and inflating them by a factor of about $e^{58} \approx 10^{25}$. The constant ground state energy of the compact extra dimensions is related to the dark energy (vacuum energy) driving today's accelerating expansion of our three-dimensional universe. With the usual values of $G, \hbar, c$, and Hubble constant $h_{0}=0.65$, the dark energy fraction is $\approx 0.7$. Immediately after inflation, the strong force acting only on matter was an effective "strong gravity" (about $10^{38}$ times stronger than gravity today) causing $83 \%$ of matter to coalesce into small impenetrable closed systems interacting only by gravity and constituting most of the dark matter. Other universes may have formed similarly in the infinite sweep of cosmic time, but are of no practical significance because they are profoundly elsewhere. The holographic principle (a consequence of quantum mechanics, general relativity, thermodynamics, and Shannon information theory) indicates only a finite number (about $10^{122}$ ) of bits of information encoded on the event horizon will ever be available to describe all physics within our universe. There are no sources or sinks of information outside the universe, so the universe must be described as a closed system. Matter dominance over anti-matter relates to the finite number of bits of information in the universe, as does the fact that theories involving continuum mathematics only approximate an underlying theory involving discrete mathematics. Ordinary matter constitutes only about $5 \%$ of the energy density of the universe. Holographic analysis of large scale structures composed of stars accounts for: minimum stellar mass as a
\end{abstract}


function of time; the hierarchy of star clusters, galaxies, and galaxy clusters; and supermassive black holes. It identifies configurations of spiral and elliptical galaxies consistent with conservation of mass and angular momentum accounting for the $15 \%$ of bulgeless disks found in the survey of 15,000 spiral galaxies in the sixth SDSS data release. The smallest scale structures are usually described by the Standard Model of elementary particles, based on local quantum field theory. The Standard Model cannot be the final theory of elementary particles because: it incorrectly assumes neutrinos are massless; it cannot account for the bizarre mass spectrum of three generations of four Standard Model fermions; and does not provide the necessary non-local discrete theory of elementary particles. A holographic (and therefore non-local) preon theory involving strands with finite energy density instead of point particles can be outlined, but calculation in such a framework is difficult and gives no insight into the fermion mass spectrum.

\section{Keywords}

Quantum Cosmology, Dark Energy, Dark Matter, Matter-Antimatter Asymmetry

\section{Introduction}

We can imagine many universes, but we only live in one characterized by three fundamental constants (gravitational constant $G=6.67 \times 10^{-8} \mathrm{~cm}^{3} / \mathrm{g} \cdot \mathrm{sec}^{2}$, Planck's constant $\hbar=1.05 \times 10^{-27} \mathrm{~g} \cdot \mathrm{cm}^{2} / \mathrm{sec}$, and speed of light $c=3.00 \times 10^{10} \mathrm{~cm} / \mathrm{sec}$ ), and governed by four forces: gravitation, electromagnetism, the strong force, and the weak interaction.

Considering cosmic time as the infinite sequence of events before and after the beginning of our universe, we may wonder about the origin of our universe. We can't experiment to replicate the origin and development of our universe. The best we can do is develop consistent and coherent explanations describing what we observe. Many such explanations may be possible. If so, we should choose the explanation with the fewest assumptions and expressed in the most convenient mathematical form. This paper describes a candidate explanation for the origin of our universe and three puzzles regarding the contents of the universe:

- why the universe contains a mysterious dark energy (vacuum energy), exerting negative pressure accelerating the expansion of the universe,

- why most matter in the universe is an equally mysterious dark matter we cannot see, and

- why anti-matter is rare.

\section{Background}

\subsection{Mathematical Basis}

- Quantum mechanics, explaining discontinuous changes we observe at very 
small distances.

- General relativity, describing large scale aggregations of matter and energy in terms of dynamics of space itself and predicting black holes.

- Thermodynamics, dealing with relations between heat, temperature, energy and work.

- Shannon information theory, describing information in terms of $(0,1)$ binary bits.

\subsection{Time}

The time variable marking the sequence of events before and after the beginning of our universe extends into the infinite past, allowing for quantum fluctuations occurring before the origin of our universe. It is identified with the time variable in quantum mechanics and the cosmic time in our Friedmann universe.

\subsection{Force Unification and Fundamental Scales in the Universe}

This explanation assumes the four basic forces acting in the universe were once united, so gravity was initially much stronger. When the symmetry between gravity and the strong-electroweak interaction broke, the changing strength of gravity resulted in inflation. Today, the gravitational structure constant $\frac{G m_{p}^{2}}{\hbar c}=5.91 \times 10^{-39}$ is the ratio of the strength of gravity to that of the strong force, and the Planck mass $M_{P}=\sqrt{\frac{\hbar c}{G}}=2.17 \times 10^{-5} \mathrm{~g}$. Initially, gravity and the unified strong-electroweak force had equal strength, and $\frac{G m_{p}^{2}}{\hbar c}=1$. The gravitational constant was initially $G_{i}=\left(\frac{M_{P}}{m_{p}}\right)^{2} G=1.69 \times 10^{38} G$, so $\frac{G m_{p}^{2}}{\hbar c}=\frac{G M_{P}^{2}}{\hbar c}=1$, the Planck length was $\delta_{i}=\sqrt{\frac{\hbar G_{i}}{c^{3}}}=\left(\frac{M_{P}}{m_{p}}\right) \delta=2.11 \times 10^{-14} \mathrm{~cm}$, and the Planck mass was $M_{P_{i}}=\sqrt{\frac{\hbar c}{G_{i}}}=m_{p}$.

\subsection{Inflation}

All observations indicate our universe began about 14 billion years ago in a hot, dense initial state and has expanded ever since. Today, the universe is very uniform, even at points so far apart that signals traveling at the speed of light (the fastest any signal can travel in our universe) could never connect them in the time since the Big Bang. The uniformity results from inflation right after the beginning of the universe, when the size of the universe increased faster than the speed of light, with distances increasing by a factor of about $10^{26}$. During inflation, the distance between points in space expanded faster than the speed of light. That does not conflict with the fact that no particle or signal moving within the universe can travel faster than light. 


\section{Quantum Theory of a Closed Universe with Three Space Dimensions}

The general relativistic Friedmann equation for the radius $R$ of a closed, homogeneous, isotropic universe with one time and three space dimensions is

$$
\left(\frac{\mathrm{d} R}{\mathrm{~d} t}\right)^{2}-\left(\frac{8 \pi G}{3}\right) \varepsilon\left(\frac{R}{c}\right)^{2}=-c^{2}
$$

where energy density of the universe $\varepsilon=\varepsilon_{r}\left(\frac{R_{0}}{R}\right)^{4}+\varepsilon_{m}\left(\frac{R_{0}}{R}\right)^{3}+\varepsilon_{v}$ and $\varepsilon_{r}$, $\varepsilon_{m}, \varepsilon_{v}$ and $R_{0}$ are, respectively, today's values of radiation, matter, and vacuum energy densities and the radius of the universe. Multiplied by $\frac{m}{2}$, the Friedmann equation becomes the following equation of motion for a fictitious particle with mass $m$ and energy $-\frac{m}{2} c^{2}$

$$
\frac{m}{2}\left(\frac{\mathrm{d} R}{\mathrm{~d} t}\right)^{2}-\frac{m}{2}\left(\frac{8 \pi G}{3}\right)\left[\varepsilon_{r}\left(\frac{R_{0}}{R}\right)^{4}+\varepsilon_{m}\left(\frac{R_{0}}{R}\right)^{3}+\varepsilon_{v}\right]\left(\frac{R}{c}\right)^{2}=-\frac{1}{2} m c^{2}
$$

Vacuum energy drove inflation, when the universe expanded almost instantaneously by about 26 orders of magnitude soon after it began. After inflation, $\varepsilon_{r}=4.4 \times 10^{-34} \mathrm{~g} / \mathrm{cm}^{3} c^{2}, \varepsilon_{m} \approx \varepsilon_{v} \approx 10^{-30} \mathrm{~g} / \mathrm{cm}^{3} c^{2}$ and $R_{0} \approx 10^{28} \mathrm{~cm}$. The radiation term in the potential dominated in the early universe, when $R \ll 10^{-5} R_{0}$, and matter and vacuum energy densities can be neglected in the early universe.

Attempts to unify general relativity and quantum mechanics are complicated by the fact that general relativity is a theory of space while quantum mechanics is a theory written in space. However, Elbaz and Novello [1] showed the equation of motion above, resulting from the Friedmann equation, can be quantized just as in any first course in quantum mechanics. The solution of the resulting Schrodinger equation provides a quantum theory of space describing evolution of a closed, homogeneous, isotropic universe like ours, with three space dimensions.

\section{The Holographic Principle Requires a Closed Universe}

General relativity is a classical theory, based on continuum mathematics, inevitably involving an infinite number of degrees of freedom. General relativity allows for finite (closed) universes and infinite (flat and open) universes. In contrast, we've long known we live in a quantized universe, so it will eventually be necessary to unify quantum mechanics and general relativity. The holographic principle is a step towards combining quantum mechanics and general relativity. It combines basic concepts of horizons and black holes from general relativity with thermodynamics, Shannon information theory, and quantum mechanics to conclude that, in a vacuum dominated universe like ours, only a finite number (about $5 \times 10^{122}$ ) of bits of information will ever be able to describe our universe [2]. Since only a finite amount of information will ever be available to describe 
the universe and there are no sources or sinks of information outside the universe, the universe must be considered a closed system described by discrete mathematics with a finite number of degrees of freedom. Flat and open universes, infinite in extent and allowed by the continuum mathematics of "classical" general relativity, are irrelevant options, even aside from the logical difficulties associated with instantaneous appearance of a universe with infinite extent at the moment of the Big Bang.

The holographic principle says all physics at any point in our universe is described by the finite number of bits of information on the horizon from which a light signal could ever reach the point. In an expanding universe such as ours, all of the universe was once within the radius of today's event horizon. So, in a closed universe, with no source or sink of information outside the universe, the total amount of information in the universe must be constant, and the total information available on the event horizon must be the constant total amount of information available to describe the entire universe, within and outside the event horizon, throughout the existence of the universe. The holographic principle also relates thermodynamics of bits of information on a horizon to general relativity as the appropriate theory of gravity within the horizon [3].

If $H_{0}=65 \mathrm{~km} \cdot \mathrm{sec}^{-1} \cdot \mathrm{Mpc}^{-1}$, the constant vacuum energy density $\varepsilon_{v}$ in our universe relates to a cosmological constant $\Lambda$ by $\Lambda=\frac{8 \pi G \varepsilon_{v}}{c^{4}} \approx 1 \times 10^{-56} \mathrm{~cm}^{-2}$. As radius $R$ of the universe increases, radiation and matter energy density will be driven to zero by expansion of the universe. The universe will act as a vacuum solution of Einstein's equations with positive cosmological constant and event horizon at distance $R_{H}=\sqrt{\frac{3}{\Lambda}}=1.7 \times 10^{28} \mathrm{~cm}$ from any observer. In a universe with an event horizon, no events beyond distance $R_{H}$ can ever be observed. The holographic principle says all information that will ever be available about physics within the event horizon is the finite number of bits of information on the event horizon, specified by one quarter of event horizon area in Planck units. Therefore, only $N_{\text {bits }}=\pi R_{H}^{2} /\left(\delta^{2} \ln 2\right) \approx 5 \times 10^{122}$ bits of information will ever be available to describe the location of matter within the universe. Thus, the universe must be a closed system containing all information it began with, with no sources or sinks of information outside the universe. Theories involving continuum mathematics, like quantum field theory (with its associated singularities and infinities), can only approximate an underlying finite-dimensional holographic theory based on discrete mathematics.

The radius $R_{0}$ of our closed universe today can be estimated using the holographic principle and two mass scales (identified by Paul Wesson [4] and involving $G, \hbar, \mathcal{c}$, and the cosmological constant $\Lambda$ ) with their geometric mean equal to the Planck mass. Introducing a proportionality constant $\xi$, Wesson's mass scales are a small mass scale $m_{b}=\left(\frac{\hbar}{c}\right) \sqrt{\frac{\Lambda}{\xi}}$ and a large mass scale 
$m_{u}=\left(\frac{c^{2}}{G}\right) \sqrt{\frac{\xi}{\Lambda}}$, with geometric mean $\sqrt{m_{u} m_{b}}=M_{P}$. Identify the large mass scale $m_{u}$ with the estimated total conserved quantity of matter in our closed vacuum-dominated universe, and the small mass scale $m_{b}$ with the estimated smallest mass in the universe (that associated with a bit of information describing matter distribution in the universe). That allows an estimate, consistent with Wesson's idea, of today's radius of the universe. With $\sqrt{m_{u} m_{b}}=M_{P}$, $\frac{m_{u}}{M_{P}}=\frac{M_{P}}{m_{b}}=k$, where $k$ is a constant. Since $m_{u}=k M_{P}$ and $M_{P}=k m_{b}$, $m_{u}=k^{2} m_{b}$ and $k^{2}=N$ where $N$ is the total number of bits of information available to describe physics within the event horizon. The two equations $m_{u}=\frac{c^{2}}{G} \sqrt{\frac{\xi}{\Lambda}}=\sqrt{N} \sqrt{\frac{\hbar c}{G}}$ and $m_{b}=\frac{\hbar}{c} \sqrt{\frac{\Lambda}{\xi}}=\frac{1}{\sqrt{N}} \sqrt{\frac{\hbar c}{G}}$ each require $\xi=\frac{3 \pi}{\ln (2)}$.

The total conserved amount of matter in the universe $m_{u}$ and $m_{b}$, the mass associated with a bit of information, can be estimated by parameterizing today's radius of the universe as $R_{0}=\beta c / H_{0}$. If matter fraction of total energy density in the universe today is $f_{m}=0.30$, total conserved amount of matter in the universe is $m_{u}=2 \pi^{2} R_{0}^{3} \rho_{m}=2 \pi^{2}\left(\frac{\beta c}{H_{0}}\right)^{3} f_{m}\left(\frac{3 H_{0}^{2}}{8 \pi G}\right)=\frac{3 \pi}{4} f_{m} \frac{\beta^{3} c^{3}}{G H_{0}}$. Mass associated with a bit of information in the universe is $m_{b}=\frac{m_{u}}{N}=\beta^{3} f_{m}\left(1-f_{m}\right) \frac{3 \ln (2)}{4} \frac{\hbar H_{0}}{c^{2}}$. Setting $\sqrt{m_{u} m_{b}}=M_{P} \quad$ requires $\quad \beta=\left(\frac{16}{9 \pi \ln (2) f_{m}^{2}\left(1-f_{m}\right)}\right)^{\frac{1}{6}}=1.6$, yielding $R_{0}=2.2 \times 10^{28} \mathrm{~cm}$. Estimated $R_{0}$ exceeds the distance to the event horizon, $R_{H}=1.7 \times 10^{28} \mathrm{~cm}$ and the Schucker-Tilquin [5] lower bound on $R_{0}$, $R_{S T}=1.2 \times 10^{28} \mathrm{~cm}$.

Witten [6] noted that seven extra compact space dimensions in addition to our familiar three space dimensions are needed to accommodate

$S U(3) \times S U(2) \times U(1)$ force symmetry of the Standard Model of particle physics. The radius of these compact extra dimensions is a scalar field pervading the universe, driving inflation and accelerating expansion of the universe today.

The holographic principle indicates the number of bits of information encoded on a holographic screen of radius $r$ surrounding any point is proportional to the area of the screen. Therefore, energy per bit in Friedmann dimensions of the initial universe with radius $r_{i}$, before compact dimensions collapsed to initiate inflation, is proportional to $\frac{1}{r_{i}^{2}}$ times total energy in Friedmann dimensions. The holographic screen enclosing energy in the seven compact dimensions has area $\sim r_{i}^{6}$. Consistency with the holographic principle in Friedmann dimensions requires energy per bit of information flowing into Friedmann dimensions during inflation proportional to $\frac{1}{r_{i}^{2}}$ times total energy 
flowing in from compact dimensions. Thus, total energy in compact dimensions in the unstable initial state where $\dot{r}_{i}^{2}=0$ must be $V_{r}=k_{r} r_{i}^{4}$.

Applying the holographic principle to the initial state, the seven-dimensional subspace dominates the contribution of the three-dimensional subspace to available number of bits of information because of its higher dimensionality. Area of a seven-sphere of radius $\frac{l}{2 \pi}$ is $\frac{16 \pi^{3}}{15}\left(\frac{l}{2 \pi \delta}\right)^{6}$ in units of Planck length $\delta$. Denote surface area of the seven-dimensional subspace by $A_{7}=\frac{f 16 \pi^{3}}{15}\left(\frac{l}{2 \pi \delta}\right)^{6}$, where $f>1$ because topology of the seven-dimensional subspace is more intricate than a seven-sphere. According to the holographic principle, the number of bits of information in the seven-dimensional subspace is $N_{\text {bits }}=\frac{f 4 \pi^{3}}{15 \ln (2)}\left(\frac{l}{2 \pi \delta}\right)^{6}$. For $N_{\text {bits }}=5.1 \times 10^{122}, l=\left(1.2 \times 10^{21}\right) f^{-\frac{1}{6}} \delta$. Since $G_{i}=\left(\frac{l}{\delta}\right)^{2} G$, this indicates the nuclear force would have to be about forty-two orders of magnitude stronger than gravity to allow the initial state involving a spherical seven-dimensional subspace $(f=1)$ to contain all information needed to describe our universe. However, candidate extra dimensional spaces in theories of fundamental forces governing the universe are far more complex than a sphere (for example, Calabi-Yau manifolds). If the perimeter of planar sections of the seven-dimensional subspace with characteristic radius $\frac{l}{2 \pi}$ is $90 l$, surface area of the subspace will be $5 \times 10^{11}$ times the area of a seven-sphere with radius $\frac{l}{2 \pi}$. Then, in eleven-dimensional space-time, initial length scale $l=2.1 \times 10^{-14} \mathrm{~cm}$ (and nuclear force $1.7 \times 10^{38}$ times stronger than gravity) produces an initial seven-dimensional subspace containing enough bits of information to describe our vacuum dominated universe.

\section{Our Closed Universe Began as a Quantum Fluctuation from Nothing}

Quantum mechanics describes discontinuous changes allowing the universe to originate in a quantum fluctuation from nothing, if (and only if) the resulting universe has all total quantum numbers equal to zero. This would not work for the closed three dimensional universe mentioned above, because quantum curvature energy $-\frac{1}{2} m c^{2}$ is non-zero in that case. Accordingly, this explanation of the origin of the universe involves seven compact space dimensions in addition to the usual four dimensions of general relativistic space-time.

Our universe began as a quantum fluctuation from nothing [7] into an unstable state of a closed ten dimensional space with total quantum numbers equal zero and curvature radius of all ten space dimensions near $\delta_{i}$. Alternatively, this unstable initial state could be reached by quantum fluctuation from a stable 
ten dimensional spherical precursor state [8] previously arising by quantum fluctuation from nothing.

The ten-dimensional curvature space has subspaces corresponding to our three dimensional Friedmann universe and seven compact dimensions, where radius of all compact dimensions is the same. When total energy and total angular momentum in curvature space are zero, the Schrodinger equation for the ten-dimensional radius is

$$
-\frac{\hbar^{2}}{2 m} \nabla_{\mathfrak{R}}^{2} \Psi+V_{\mathfrak{R}} \Psi=0
$$

where $\mathfrak{R}$ is the magnitude of a ten dimensional vector and $m$ is an effective mass.

The initial state had zero total energy with $\mathfrak{R}^{2}=R^{2}+r^{2}, R$ the radius of curvature of the three-dimensional isotropic subspace of our Friedmann universe, and $r$ the radial coordinate in the seven-dimensional subspace describing curvature of compact dimensions. In this state, $\sqrt{\left\langle R^{2}\right\rangle}=\sqrt{\left\langle r^{2}\right\rangle}=\frac{\delta_{i}}{2 \pi}$ and $\dot{R}=\dot{r}=0$ at the beginning of our universe. The size of the compact dimensions corresponds to a gauge singlet scalar field, constant throughout the three dimensional Friedmann universe, that inflated the early universe and is responsible for the vacuum energy (dark energy) dominating the universe today.

The potential describing the three dimensional Friedmann universe and the compact subspace in our universe is $V_{\mathfrak{R}}=V_{R}+V_{r}$, where $V_{r}=k_{r} r^{4}$. With $V_{\mathfrak{R}}=V_{R}+V_{r}, \Psi(\Re)=\Psi(R) \Psi(r)$ and

$$
\begin{aligned}
& {\left[\frac{1}{\psi(R)} \frac{-\hbar^{2}}{2 m} \nabla_{R}^{2} \psi(R)+V_{R}\right]} \\
& +\left[\frac{1}{\psi(r)} \frac{-\hbar^{2}}{2 m} \nabla_{r}^{2} \psi(r)+V_{r}\right]=0
\end{aligned}
$$

where each bracket is a constant, denoted $-E$ and $E$ respectively. So, curvature energy of the closed three dimensional Friedmann universe is $E_{c 3}=-E$, curvature energy of the seven compact dimensions is $E_{c 7}=E$, and total curvature energy of the universe is $E_{c t o t}=E_{c 3}+E_{c 7}=0$. The universe evolved from this excited state with large curvature energies $-E$ and $E$ (where $|E| \gg \frac{m c^{2}}{2}$ ) into today's universe where curvature energy of the three dimensional Friedmann universe has the Einstein value $-\frac{m c^{2}}{2}$, and compact dimensions are in the ground state of the potential $V_{r}$, with curvature energy $\frac{m c^{2}}{2}$. An S-wave Schrodinger equation must be used for compact dimensions to make total ten-dimensional "angular momentum" in the curvature space zero. With $\Psi=R^{-1} \psi(R) r^{-3} \psi^{\prime}(r)$, the separated Schrodinger equation becomes 


$$
\begin{aligned}
& {\left[\frac{1}{\psi(R)} \frac{-\hbar^{2}}{2 m} \frac{\mathrm{d}^{2}}{\mathrm{~d} R^{2}} \psi(R)+V_{R}\right]} \\
& +\left[\frac{1}{\psi^{\prime}(r)} \frac{-\hbar^{2}}{2 m} \frac{\mathrm{d}^{2}}{\mathrm{~d} r^{2}} \psi^{\prime}(r)+\left(\frac{3 \hbar^{2}}{m r^{2}}+V_{r}\right)\right]=0
\end{aligned}
$$

\section{Dark Energy Relates to Ground State Energy of Compact Extra Dimensions}

Inflation occurred during collapse of seven compact dimensions from a size near $\delta_{i}$ to their ground state with size near the Planck length $\delta$. Dark energy (vacuum energy) fraction $\Omega_{\Lambda}$ in the inflated universe relates to ground state energy of the seven compact extra dimensions. Details of the straightforward calculation estimating dark energy fraction as $\Omega_{\Lambda} \approx 0.7$ are in the Appendix.

\section{Dark Matter}

Immediately after inflation, the strong force acting only on matter was an effective "strong gravity" with strength $G_{S}=\left(\frac{M_{P}}{m_{p}}\right)^{2} G=1.7 \times 10^{38} G$. The strong gravity Friedmann equation $\left(\frac{\mathrm{d} R}{\mathrm{~d} t}\right)^{2}-\frac{8 \pi G_{S} \rho R^{2}}{3}=-c^{2}$ described local curvature of spaces defining closed massive systems bound by the effective strong gravity. Because strong force at short distances is involved, quantum mechanical analysis of such systems is necessary [9]. The Schrodinger equation resulting from Elbaz-Novello quantization of the Friedmann equation for closed massive systems bound by effective strong gravity is $-\frac{\hbar^{2}}{2 \mu} \frac{\mathrm{d}^{2}}{\mathrm{~d} r^{2}} \psi-\frac{2 G_{S} \mu M}{3 \pi r} \psi=-\frac{\mu c^{2}}{2} \psi$, where $M=2 \pi^{2} \rho r^{3}$ is the conserved mass of closed systems with radius $r$ and $\mu$ is an effective mass. This Schrodinger equation is identical in form to the Schrodinger equation for the hydrogen atom and can be solved immediately. Ground state curvature energy $-\frac{\mu}{2 \hbar^{2}}\left(\frac{2 G_{S} \mu M}{3 \pi}\right)^{2}$ of this Schrodinger equation must equal $-\frac{\mu c^{2}}{2}$ for consistency with the corresponding Friedmann equation, so effective mass $\mu=\frac{3 \pi \hbar c}{2 G_{S} M}$. The ground state solution of this Schrodinger equation describes stable closed systems bound by effective strong gravity, with zero orbital angular momentum and radius $\langle r\rangle=\frac{G_{S} M}{\pi c^{2}}=\frac{\hbar M}{\pi c m_{p}^{2}}$. Geodesic paths inside the stable ground state closed systems created by effective strong gravity are all circles with radius $\langle r\rangle=\frac{\hbar M}{\pi c m_{p}^{2}}$, so matter within these closed systems is permanently confined within a sphere of radius $\langle r\rangle$. No particle can enter or leave the small closed systems after they form, to increase or decrease the 
amount of matter in those closed systems.

Assuming velocity-independent rigid sphere scattering, the (self-interaction collision cross-section)/mass ratio for dark matter particles is $\frac{\sigma}{M}=\frac{4 \pi(2 r)^{2}}{M}$. Consider values of $\frac{\sigma}{M}$ between $0.015 \mathrm{~cm}^{2} / \mathrm{g}$ and $0.025 \mathrm{~cm}^{2} / \mathrm{g}$, rounding to Hennawi and Ostriker's [10] estimate of $\frac{\sigma}{M}=0.02 \mathrm{~cm}^{2} / \mathrm{g}$. Inserting dark matter particle radius/mass relation $r=\frac{\hbar M}{\pi c m_{p}^{2}}$ into rigid sphere (self-interaction collision cross-section)/mass relation $\frac{\sigma}{M}=\frac{4 \pi(2 r)^{2}}{M}$ yields $M=\left[\left(\frac{\sigma}{M}\right) \frac{\pi}{16}\left(\frac{c}{\hbar}\right)^{2} m_{p}^{3}\right] m_{p}$. Values of $\frac{\sigma}{M}$ between $0.015 \mathrm{~cm}^{2} / g$ and 0.025 $\mathrm{cm}^{2} / \mathrm{g}$ indicate dark matter particle mass between $10.5 \mathrm{GeV}$ and $17.5 \mathrm{GeV}$ and radius $r=\left(\frac{A}{\pi}\right)\left(\frac{\hbar}{m_{p} c}\right)$ between $0.75 \mathrm{fm}$ and $1.25 \mathrm{fm}$.

If $\frac{\sigma}{M}=0.02 \mathrm{~cm}^{2} / \mathrm{g}$, dark matter particles have mass $=15 m_{p}=14 \mathrm{GeV}$, radius $=1 \mathrm{fm}$, and density $6 \times 10^{15} \mathrm{~g} / \mathrm{cm}^{3}$. As the universe expanded after inflation, matter density steadily dropped. When matter density fell to $6 \times 10^{15} \mathrm{~g} / \mathrm{cm}^{3}$, most matter coalesced into dark matter-small closed spheres with zero orbital angular momentum. This resulted in a universe packed with small invisible and impenetrable systems interacting only gravitationally. Assuming uniform matter density in the universe and instantaneous coalescence with maximum packing fraction, $74 \%$ of matter in the universe is small closed impenetrable systems constituting the bulk of dark matter. With non-uniform density, coalescence would occur first in lower density volumes, and expansion of the universe might allow slightly more than $74 \%$ of matter to coalesce into small closed systems.

Matter fraction of energy density in the universe today is about 0.3 . If hadronic matter fraction is about 0.05 , dark matter is $(0.25 / 0.3)=83 \%$ of all matter. If $74 \%$ of all matter is small closed systems, those small bound systems account for $0.74 \times 0.3=0.22$ of energy density in the universe today, or $(0.22 / 0.25)=88 \%$ of dark matter. Using $R_{0}=2.2 \times 10^{28} \mathrm{~cm}$ and today's matter density $2.4 \times 10^{-30} \mathrm{~g} / \mathrm{cm}^{3}$, matter density $6 \times 10^{15} \mathrm{~g} / \mathrm{cm}^{3}$ required for coalescence into small closed systems with mass $14 \mathrm{GeV}$ occurred when radius of the universe was $R_{c} \approx 1.6 \times 10^{13} \mathrm{~cm}$.

Impenetrable spheres of dark matter are the ultimate defense against gravitational collapse. Close-packed spheres of $n$ dark matter particles have radius $R_{n}=\sqrt[3]{n} \frac{\hbar M}{\pi c m_{p}^{2}}=\sqrt[3]{n} \mathrm{fm}$. Schwarzschild radius of those spheres, $R_{S}=\frac{29.9 G n m_{p}}{c^{2}}=3.7 n \times 10^{-51} \mathrm{~cm}$, is smaller than their physical radius until 
$\sqrt[3]{n}\left(1 \times 10^{-13} \mathrm{~cm}\right)=3.7 n \times 10^{-51} \mathrm{~cm}$, or $n=1.4 \times 10^{56}$. This indicates a minimum black holes mass of $2.1 \times 10^{57} \mathrm{~m}_{p}=3.5 \times 10^{33} \mathrm{~g}$, or about 1.5 times the solar mass, and a minimum Schwarzschild radius of $5.2 \mathrm{~km}$.

\section{Holography and Ordinary Matter-The Other $5 \%$ of the Universe}

After accounting for dark energy and dark matter, observations indicate ordinary matter we see constitutes only about $5 \%$ of total energy density of the universe.

\subsection{Rarity of Anti-Matter}

Twelve fermions are basic elements in the Standard Model of particle physics. They are obviously not "elementary", because they must eventually be described in terms of the $(+,-)$ bits of information defined by the holographic principle. So, Standard Model particles must eventually be described as composites of underlying entities called preons. Preons cannot be point particles, because they would have infinite energy density.

All Standard Model particles have charges $0,1 / 3,2 / 3$ or 1 in units of electron charge $\pm e$, so bits in a preon model must be identified with fractional electric charge. In any physical system, energy must be transferred to change information in a bit from one state to another. Labeling the low energy state of a bit $e / 3 n$ and the high energy state $-e / 3 n$ (where $n$ is an integer) defines electric charge. The universe must be charge neutral if it began by spontaneous quantum fluctuation from nothing. So, there must be equal numbers of $e / 3 n$ and $-e / 3 n$ charges. A holographic charged preon model in such a universe then embodies charge conservation, a precondition for gauge invariance and Maxwell's equations.

Protons have charge $e$ and anti-protons have charge $-e$. Therefore, regardless of how bits of information on the horizon specify a proton or anti-proton, preon configurations specifying protons must differ in $3 n$ bits from configurations specifying anti-protons. Then, because $e / 3 n$ bits and $-e / 3 n$ bits do not have the same energy, the number of protons and anti-protons created in the early universe must be slightly different. If $e / 3 n$ bits have lower energy than $-e / 3 n$ bits, there must be more matter than anti-matter in the universe [11].

Temperature at time of baryon formation was $T_{B}=2 m_{p} c^{2} / k=2.18 \times 10^{13} \mathrm{~K}$, So, radius of the universe at baryogenesis was $R_{B}=R_{0}\left(\frac{2.725}{T_{B}}\right)=2.7 \times 10^{15} \mathrm{~cm}$, where $2.725 \mathrm{~K}$ is today's cosmic microwave background temperature. Time $t_{B}$ of baryogenesis, in seconds after inflation ended, is determined from the Friedmann equation $\left(\frac{\mathrm{d} R}{\mathrm{~d} t}\right)^{2}-\left(\frac{8 \pi G}{3}\right) \varepsilon\left(\frac{R}{c}\right)^{2}=-\kappa c^{2}$. After inflation, the universe is so large, it is almost flat, and curvature parameter $\kappa \approx 0$. Energy 
density is $\varepsilon(R)=\varepsilon_{r}\left(\frac{R_{0}}{R}\right)^{4}+\varepsilon_{m}\left(\frac{R_{0}}{R}\right)^{3}+\varepsilon_{v}$, so radiation dominated when $R \ll 10^{-5} R_{0}$ before radiation/matter equality. Integrating

$$
\left(\frac{\mathrm{d} R}{\mathrm{~d} t}\right)^{2}-\left(\frac{8 \pi G}{3 c^{2}}\right) \frac{\varepsilon_{r} R_{0}^{4}}{R^{2}}=\left(\frac{\mathrm{d} R}{\mathrm{~d} t}\right)^{2}-\frac{A^{2}}{R^{2}}=0
$$

where $A=\sqrt{\frac{8 \pi G \varepsilon_{r} R_{0}^{4}}{3 c^{2}}}$, from the end of inflation at $t=0$ to $t$ gives $\frac{1}{2}\left(R^{2}-R_{i}^{2}\right)=A t$, where $R_{i}$ is radius of the universe at the end of inflation. Therefore, $t_{B}=\frac{\left(R_{B}^{2}-R_{i}^{2}\right)}{2 A} \approx \frac{R_{B}^{2}}{2 A} \approx 5 \times 10^{-7}$ seconds, if $R_{B} \gg R_{i}$. Distance $d_{B}$ from any point in the universe to the particle horizon for that point is $d_{B}=c R_{B} \int_{0}^{t_{B}} \frac{\mathrm{d} t^{\prime}}{R\left(t^{\prime}\right)}=\left[\frac{c R_{B}}{A} \sqrt{R_{i}^{2}+2 A t}\right]_{0}^{t_{B}}=\frac{c R_{B}}{A}\left[\sqrt{R_{i}^{2}+2 A t_{B}}-R_{i}\right]$. Since $R_{B} \gg R_{i}, d_{B} \approx c R_{B} \sqrt{\frac{2 t_{B}}{A}} \approx 3 \times 10^{4} \mathrm{~cm}$.

Surface gravity on the particle horizon at baryogenesis was $g_{H B}=G \frac{4 \pi}{3} \frac{\varepsilon\left(R_{B}\right)}{c^{2}} d_{B} \approx \frac{4 \pi G}{3 c} \varepsilon_{r} \frac{R_{0}^{4}}{A R_{B}^{2}}$ and associated horizon temperature was $T_{H B}=\frac{\hbar}{2 \pi c k} g_{H B} \approx 6 \times 10^{-7} \mathrm{~K} \quad[12]$. Temperature at any epoch is uniform throughout a post-inflationary homogeneous isotropic Friedman universe, and causal horizon at baryogenesis is at distance $d_{B}$ from every point in the universe. Temperature at every point on the causal horizon for every point in the universe is the same because surface gravity of the uniform sphere within the horizon is the same at every point on every horizon. Bits on all causal horizons are in thermal equilibrium, and only two quantum states are available for those bits. Therefore, equilibrium statistical mechanics can be used and occupation probabilities of the two bit states in thermal equilibrium at temperature $T_{H B}$ are proportional to their corresponding Boltzmann factors. So, if energy of an $e / 3 n$ bit on the horizon at baryon formation is $E_{b i t}-E_{d}$ and energy of a $-e / 3 n$ bit is $E_{b i t}+E_{d}$, proton/antiproton ratio at baryogenesis is $\left(e^{-\frac{E_{b i t}-E_{d}}{k T_{H B}}} / e^{-\frac{E_{b i t}-E_{d}}{k T_{H B}}}\right)^{3 n}=e^{\frac{6 n E_{d}}{k T_{H B}}}$. Since $e^{\frac{6 n E_{d}}{k T_{H B}}} \approx 1+\frac{6 n E_{d}}{k T_{H B}}$, proton excess is $\frac{6 n E_{d}}{k T_{H B}}$.

Holographic preon models must link bits of information on the horizon to bits of information specifying location of preon constituents of Standard Model particles in the universe. The wavefunction specifying probability distribution for location of a bit of information in the universe has only two energy levels. Energy released when a bit drops from $(-)$ to $(+)$ state raises another bit from $(+)$ to $(-)$ state, ensuring charge conservation. Energy must be transferred by a massless quantum with wavelength related to the size of the universe, and the only macroscopic length characteristic of the size of a closed Friedmann un- 
iverse with radius $R$ is the circumference $2 \pi R$. If the energy $2 E_{d}$ to change the state of a bit associated with a preon in the universe (and the corresponding bit on the horizon) at baryogenesis equals the energy of massless quanta with wavelength characteristic of the size of a closed Friedmann universe with radius $R_{B}, 2 E_{d}=\frac{\hbar c}{R_{B}}$. The small difference in energy of the bits of information on the horizon specifying a proton or anti-proton is consistent with protons and anti-protons having the same mass (differing by $12 E_{d} \approx 10^{-41} \mathrm{~m}_{p}$ ). Substituting from above, proton excess at baryogenesis is $\frac{6 n E_{d}}{k T_{H B}}=\left(\frac{12 n \pi c^{2}}{R_{0}}\right)\left(\frac{2.725}{T_{B}}\right) \sqrt{\frac{3}{8 \pi G \varepsilon_{r}}}$. Dependence on $R_{0}$ arises because $R_{B}$, the radius of the universe at baryogenesis, depends on $R_{0}$, today's cosmic microwave background temperature $2.725 \mathrm{~K}$, and temperature $T_{B}$ at baryogenesis.

If $R_{0}=2.2 \times 10^{28} \mathrm{~cm}$, proton excess is $\frac{6 n E_{d}}{k T_{H B}}=0.4 n \times 10^{-9}$. For comparison, estimated ratio of baryon density to cosmic microwave background photon density is $0.6 \times 10^{-9}$ [13]. If preons are strands of finite length, strand ends can be characterized by $\mathrm{a}+$ or - bit identified with electric charge. If $n=2$, each strand end has charge $\pm e / 6$, and one charged strand plus two neutral strands can describe a particle with charge $e / 3$. Three strands with charges $\pm e / 6$ on their ends are necessary and sufficient to produce entities corresponding to particles of the Standard Model with no room for additional particles besides those in the Standard Model. So preon configurations with $n>2$ are unnecessary.

\subsection{Holography and Large Scale Structure}

The holographic principle accounts for [14]: minimum stellar mass at various ages of the universe; development of large scale structures composed of stars and including star clusters, galaxies and galaxy clusters today; and existence of supermassive black holes. It also identifies configurations of spiral and elliptical galaxies consistent with conservation of mass and angular momentum, accounting for the 15\% of bulgeless disks found among 15,000 spiral galaxies in the sixth SDSS data release [15].

\subsection{Holography and Smallest Scale Structures}

The Standard Model of particle physics can only approximate an underlying finite-dimensional non-local theory involving fundamental entities that are not point particles. First, the Standard Model is a local field theory, and (consistent with quantum mechanics) there are non-local effects in our universe [16]. Second, the holographic principle indicates only a finite number of bits of information will ever be available to describe the observable universe. Third, energy densities of Standard Model point particles are infinite, and infinities in physical theories indicate inadequacies of those theories. In contrast, a non-local 
model [17], involving preon strands with finite energy density instead of point particles, links $(+,-)$ bits of holographic information to Standard Model particles.

Quantum mechanics is non-local [16], and a holographic quantum mechanical theory is essentially non-local if the wavefunction on the horizon (describing evolution of all information available about the observable universe) is the boundary condition on the wavefunction describing information distribution within the horizon. Then any quantum transition altering the wavefunction on the horizon is reflected in an instantaneous non-local change in the wavefunction describing distribution of information within the horizon and thus describing all physics in the observable universe.

\section{Falsification}

All or part of the explanation outlined above must be abandoned if there is convincing evidence that

- cosmic inflation did not occur;

- dark energy is not constant in space and time;

- dark matter interacts weakly, annihilates, or is not well described as small impenetrable spheres. (Note that scattering of neutral hydrogen off the small impenetrable spheres of dark matter will inevitably cool the hydrogen gas, to an extent on the order of that observed at $z=17$ by EDGES in the Western Australian outback);

- a simpler explanation is found for matter dominance over anti-matter; or

- black holes with mass $<1.5 M_{\odot}$ are found.

\section{References}

[1] Elbaz, E., Novello, M., Salim, J., Motta da Silva, M. and Klippert, R. (1997) General Relativity and Gravitation, 29, 481.

Novello, M., Salim, J., Motta da Silva, M. and Klippert, R. (1996) Physical Review D, 54,6202 .

[2] Bousso, R. (2002) Reviews of Modern Physics, 74, 825. https://doi.org/10.1103/RevModPhys.74.825

[3] Padmanabhan, T. (2014) General Relativity and Gravitation, 46, 1673. https://doi.org/10.1007/s10714-014-1673-7 Padmanabhan, T. (2016) Exploring the Nature of Gravity. arXiv:1602.01474 [gr-qc]

[4] Wesson, P. (2004) Modern Physics Letters A, 19, 1995. https://doi.org/10.1142/S0217732304015270

[5] Schucker, T. and Tilquin, A. (20005) From Hubble Diagrams to Scale Factors. arXiv:astro-ph/0506457

[6] Witten, E. (1981) Nuclear Physics B, 186, 412-428. https://doi.org/10.1016/0550-3213(81)90021-3

[7] Mongan, T. (2001) General Relativity and Gravitation, 33, 1415-1424. https://doi.org/10.1023/A:1012065826750

[8] Mongan, T. (2005) General Relativity and Gravitation, 37, 967-970. https://doi.org/10.1007/s10714-005-0079-y 
[9] Mongan, T. (2011). Dark Matter from "Strong Gravity”-Consistent with CRESST, CoGeNT and DAMA/LIBRA. arXiv:0706.3050 [gr-qc]

[10] Hennawi, J. and Ostriker, J. (2002) The Astrophysical Journal, 572, 41. https://doi.org/10.1086/340226

[11] Mongan, T. (2012) Holography, Charge and Baryon Asymmetry. arXiv:0911.3660 [physics.gen-ph]

[12] Padmanabhan, T. (2010) AIP Conference Proceedings, 1241, 93-108. Padmanabhan, T. (2009) A Dialogue on the Nature of Gravity. arXiv:0910.0839 [gr-qc]

[13] Bennet, C., et al. (2003) The Astrophysical Journal Supplement Series, 1, 175.

[14] Mongan, T. (2015) Holography, Large Scale Structure, Supermassive Black Holes, and Minimum Stellar Mass. arXiv:1301.0304 [physics.gen-ph]

[15] Mongan, T. (2017) JMP, 8, 2179-2188. https://doi.org/10.4236/jmp.2017.814133

[16] Aspect, A., et al. (1982) Physical Review Letters, 49, 1804. https://doi.org/10.1103/PhysRevLett.49.1804

[17] Mongan, T. (2013) A Holographic Charged Preon Model. arXiv:0801.3670 [physics.gen-ph] 


\section{Appendix: Estimating Dark Energy Fraction as $\Omega_{\Lambda} \approx 0.7$}

Our universe involves an effective potential $V_{r}=k_{r} r^{4}$ in the seven compact dimensions with a minimum near $\frac{\delta}{2 \pi}$. If $k_{r}=\frac{3 \hbar^{2}}{2 m}\left(\frac{2 \pi}{f_{7} \delta}\right)^{6}$ the minimum of the effective potential in the compact dimensions is at $r=\frac{f_{7} \delta}{2 \pi}$, where $f_{7}$ ensures radius of the compact dimensions today is $\sqrt{\left\langle r^{2}\right\rangle}=\frac{\delta}{2 \pi}$. Approximating $V_{r}$ by a harmonic oscillator potential near its minimum, ground state curvature energy of compact dimensions is $E_{g}=\frac{1}{2 m}\left(\frac{2 \pi \hbar \beta_{7}}{f_{7} \delta}\right)^{2}$, where $\beta_{7}=\sqrt{15}$. Setting $E_{g}=\frac{m c^{2}}{2}$ establishes effective mass as $m=\left(\frac{2 \pi \beta_{7}}{f_{7}}\right) M_{P}$, so $k_{r}=\frac{3 M_{P} c^{2}}{4 \beta_{7} \delta^{4}}\left(\frac{2 \pi}{f_{7}}\right)^{5}$.

Ground state wavefunction of the compact dimensions is $\psi^{\prime}(r) \sim e^{-3\left(\frac{2 \pi r}{f_{7}}-1\right)^{2}}$. With $x=r-y$ and $y=\frac{f_{7} \delta}{2 \pi}$,

$$
\left\langle r^{2}\right\rangle=\left(\int_{-y}^{\infty}(x+y)^{2} e^{-3\left(\frac{x}{y}\right)^{2}} \mathrm{~d} x\right) /\left(\int_{-y}^{\infty} e^{-3\left(\frac{x}{y}\right)^{2}} \mathrm{~d} x\right)
$$

or (correcting two mis-prints in arXiv:gr-qc/0103021)

$$
\left\langle r^{2}\right\rangle=\left(\frac{f_{7} \delta}{2 \pi}\right)^{2}\left(\frac{e^{-3}}{\sqrt{3}}+\frac{7 \sqrt{\pi}}{6}[1+\operatorname{Erf}(\sqrt{3})]\right) /(\sqrt{\pi}[1+\operatorname{Erf}(\sqrt{3})])
$$

where Erf is the error function. Then $\sqrt{\left\langle r^{2}\right\rangle}=\frac{\delta}{2 \pi}$ if

$$
f_{7}=\left\{\left(\frac{e^{-3}}{\sqrt{3}}+\frac{7 \sqrt{\pi}}{6}[1+\operatorname{Erf}(\sqrt{3})]\right) /(\sqrt{\pi}[1+\operatorname{Erf}(\sqrt{3})])\right\}^{-\frac{1}{2}}=0.92
$$

Just prior to inflation, radius of all dimensions of the universe was $\frac{\delta_{i}}{2 \pi}$. Compact dimensions were in a highly excited state of the effective potential $V_{r}$, with wave packet localized at classical turning radius $r=\frac{\delta_{i}}{2 \pi}$, and curvature energy $E^{\prime}=\frac{3 \pi M_{p} c^{2}}{\beta_{7} f_{7}^{5}}\left(\frac{\delta_{i}}{\delta}\right)^{4}$. Curvature energy in Friedmann dimensions just before inflation, $-E^{\prime}$, coincided with the top of the effective potential

$$
V_{\phi}=-\frac{4 \pi m_{\phi} G_{\phi}}{3}\left(\varepsilon_{r}+\varepsilon_{\phi}\right)\left(\frac{R}{C}\right)^{2}=-\frac{4 \pi \delta_{\phi}}{3}\left(\frac{A^{\prime}}{R^{2}}+\varepsilon_{\phi} R^{2}\right)
$$

in the separated Schrodinger equation for the wave function $\psi(R)$ of a Friedmann universe containing radiation and a scalar field. The effective potential is 
the potential for a radiation dominated universe when $R \rightarrow 0$ or $\varepsilon_{r} \gg \varepsilon_{\phi}$. The scalar field $\phi$ corresponds to the size of the compact dimensions. Scalar field energy density $\varepsilon_{\phi}=\dot{\phi}^{2}+V_{\phi}(\phi)$ is constant throughout space, but decreased rapidly during inflation. Subscript $\phi$ indicates that $G=G_{\phi}$, effective mass $m_{\phi}$, Planck length $\delta_{\phi}$, and scalar field energy density $\varepsilon_{\phi}$ depend on the value of the scalar field $\phi$ and changed almost instantaneously as compact dimensions collapsed and inflated our familiar three space dimensions. Initial state wave packet was centered at $R_{\text {peak }}=\frac{\delta_{i}}{2 \pi}$, in unstable equilibrium, with $\dot{R}=0$ and $\varepsilon_{\phi}=A^{\prime}\left(\frac{2 \pi}{\delta_{i}}\right)^{4}$, so $\varepsilon_{r}=\varepsilon_{\phi}$ and $\frac{8 \pi}{3} m_{\phi} G_{\phi} A^{\prime}\left(\frac{2 \pi}{c \delta_{i}}\right)^{2}=\frac{3 \pi M_{P} c^{2}}{\beta_{7} f_{7}^{5}}\left(\frac{\delta_{i}}{\delta}\right)^{4}$.

When curvature energy of compact dimensions dropped to the ground state energy $E_{g}=\left(\frac{\pi \beta_{7}}{f_{7}}\right) M_{P} c^{2}$, curvature energy of our three dimensions was raised to $-E_{g}=-\left(\frac{\pi \beta_{7}}{f_{7}}\right) M_{P} c^{2}$. The scalar field changed from its initial value $\phi_{i}$ to its present value $\varphi_{f}$ as characteristic size of compact dimensions decreased from $\delta_{i}$ to $\delta, G$ decreased from $G_{i}$ to its present value, and Planck mass increased from the proton mass $m_{p}$ to its present value $M_{P}=\sqrt{\frac{\hbar c}{G}}=2.17 \times 10^{-5} \mathrm{~g}$.

Inflation occurred as radius of the compact dimensions shrank from $\delta_{i}$ to $\delta$ and curvature energy of compact dimensions dropped from $E^{\prime}$ to the ground state energy $E_{g}$, raising curvature energy of Friedmann dimensions to $-E_{g}$. Entropy was injected into the scalar field in Friedmann dimensions and transferred to radiation as the scalar field decayed during inflation. When $R>R_{\text {peak }}$, the $\varepsilon_{\phi}$ term in the effective potential for $\psi(R)$ dominated and Friedmann dimensions inflated.

Just before inflation, $\dot{r}=0, \dot{\phi}=0$ and scalar field energy density was $\varepsilon_{\phi}=\dot{\phi}^{2}+V_{\phi}\left(\phi_{i}\right)=V_{\phi}\left(\phi_{i}\right)$. When compact dimensions reached their ground state at the end of inflation, $\left\langle\dot{r}^{2}\right\rangle=\left\langle\dot{\phi}^{2}\right\rangle=0$ thereafter, and scalar field energy density remained constant at $\varepsilon_{\phi}=\dot{\phi}^{2}+V_{\phi}\left(\phi_{f}\right)=V_{\phi}\left(\phi_{f}\right)=\varepsilon_{v}$, the vacuum energy today (related to the cosmological constant $\Lambda$ by $\Lambda=\frac{8 \pi G}{c^{4}} \varepsilon_{v}$ ). After scalar field energy stopped decaying to radiation at the end of inflation, the energy conservation equation separated into two parts, one for radiation and one for scalar field. Then scalar field pressure is $p_{\phi}=\dot{\phi}^{2}-V_{\phi}\left(\phi_{f}\right)=-V_{\phi}\left(\phi_{f}\right)=-\varepsilon_{\phi}=-\varepsilon_{v}$ and like scalar field energy density it remained constant thereafter. At the end of inflation, after the scalar field stopped decaying to radiation, $\varepsilon_{\phi} \ll \varepsilon_{\text {radiation }}$, and Schrodinger wave equation for radius of the radiation-dominated universe was

$$
-\frac{\hbar^{2}}{2 m} \frac{\mathrm{d}^{2}}{\mathrm{~d} R^{2}} \psi-\frac{m G}{2} \frac{A}{R^{2}} \psi=-\frac{m c^{2}}{2} \psi
$$

with $A=\left(\frac{3}{\left(2 \pi \beta_{7}\right)^{2} f_{7}^{4}}\right) \frac{\hbar}{c}\left(\frac{\delta_{i}}{\delta}\right)^{6}$. Then, $V_{\text {radiation }}=8.3 \frac{m}{2} \frac{10^{67}}{R^{2}}$, and calculated 
radiation density is $\varepsilon_{r}=6.6 \times 10^{-40} \mathrm{~g} / \mathrm{cm}^{3} c^{2}$ if $R_{0}=2.2 \times 10^{28} \mathrm{~cm}$. However, nuclear energy levels, determined by the strong force, are $\sim 10^{6}$ times atomic energy levels determined by the electromagnetic force, so strong-electroweak symmetry breaking increased initial radiation density of the universe by about six orders of magnitude. When some primordial radiation decayed to hadrons, remaining photons resulted in today's microwave background radiation energy density $\varepsilon_{r}=4.4 \times 10^{-34} \mathrm{~g} / \mathrm{cm}^{3} c^{2}$.

The extent of inflation at the beginning of the universe is estimated (see figure 1 in Ref. [7]) by assuming curvature energy of compact dimensions dropped instantaneously to ground state energy when radius of compact dimensions reached $\langle r\rangle=\frac{\delta}{2 \pi}$. The ratio $\frac{E^{\prime}}{E_{g}}=\frac{3}{2 \beta_{7}^{2} f_{7}^{4}}\left(\frac{\delta_{i}}{\delta}\right)^{4}=6.41 \times 10^{75}$. At the end of inflation, compact dimensions were in their ground state and could no longer transfer energy to Friedmann dimensions. The scalar field had decayed to radiation and could no longer transfer entropy to the radiation field. Assuming curvature energy of compact dimensions dropped instantaneously to the ground state when radius of compact dimensions reached $\langle r\rangle=\frac{\delta}{2 \pi}$, entropy of compact dimensions was reduced by a factor of $0.13 \times 10^{-75}$ and entropy of the scalar field in the Friedmann universe was increased by a factor of $6.41 \times 10^{75}$ at the beginning of inflation. If $T_{0}$ is temperature of the Friedmann universe at the end of inflation, when entropy in the scalar field stopped being transferred to radiation, temperature of the scalar field at the beginning of inflation was $\left(6.41 \times 10^{75}\right)^{\frac{1}{3}} T_{0}=1.86 \times 10^{25} T_{0}$. During isentropic expansion, $R T$ remains constant. So, injection of entropy from collapse of compact dimensions increased the radius of the three space dimensions of our Friedmann universe by a factor of $1.86 \times 10^{25}$ as the Friedmann universe expanded exponentially and isentropically (driven by the scalar field $\phi$ ), until inflation ended when $\dot{\phi}=0$ and temperature of the universe was $T_{0}$. This 58 e-fold inflation is within limits set by fluctuations in microwave background radiation. When strong-electroweak symmetry broke during inflation, temperature in Friedmann dimensions in creased by a factor $\sim\left(10^{6}\right)^{\frac{1}{3}}$ at some instant during inflation. This increased inflation of Friedmann dimensions by a factor $\sim\left(10^{6}\right)^{\frac{1}{3}}=e^{4.6}$ for total inflation of more than 60 e-folds.

Vacuum energy density today is scalar field energy density per unit coordinate volume (i.e., vacuum energy density is scalar field energy density per unit coordinate volume multiplied by the number of unit coordinate volumes in the universe and divided by volume of the universe in unit coordinate volumes). Vacuum energy density has been constant since the scalar field decoupled from the radiation field at the end of inflation. It is the energy density associated with creation of one cubic centimeter of space during expansion of the universe. Scalar 
field energy density in the co-moving volume of the universe at the beginning of inflation can be found from the Schrodinger wave equation

$-\frac{\hbar^{2}}{2 m_{\phi}} \frac{\mathrm{d}^{2}}{\mathrm{~d} R^{2}} \psi-\frac{4 \pi \delta_{\phi}}{3}\left(\varepsilon_{r}+\varepsilon_{\phi}\right) R^{2} \psi=-E^{\prime} \psi$ for the radius $R$ of the Friedmann dimensions just before inflation, when $\dot{R}=0$ and the radius $R=\frac{\delta_{\phi}}{2 \pi}=\frac{\delta_{i}}{2 \pi}$. Since $\varepsilon_{r}=\varepsilon_{\phi}$ at transition, $\frac{4 \pi \delta_{i}}{3}\left(2 \varepsilon_{\phi}\right)\left(\frac{\delta_{i}}{2 \pi}\right)^{2}=E^{\prime}$. This scalar field energy density $\varepsilon_{\phi}$ is total scalar field energy in the three-sphere with radius $R=\frac{\delta_{i}}{2 \pi}$ comprising the initial Friedmann universe, divided by volume of the three-sphere. It is related to $\eta_{\phi}$, scalar field energy density per unit coordinate volume at the beginning of inflation, by $\varepsilon_{\phi}=\frac{1}{2 \pi^{2}}\left(\frac{2 \pi}{\delta_{i}}\right)^{3} \eta_{\phi}$. So, energy density per unit coordinate volume of the scalar field in the Friedmann universe at the start of inflation was $\eta_{\phi}=5.90 \times 10^{92} \mathrm{~g} \cdot \mathrm{cm}^{-1} \cdot \mathrm{sec}^{-2}$. A spatially constant scalar field has only one degree of freedom, and energy density in the scalar field is proportional to the fourth power of temperature. So, energy density per unit coordinate volume of the scalar field at the end of inflation (vacuum energy density in our universe today), is $\eta_{e}=\frac{T_{0}^{4}}{\left(1.86 \times 10^{25} T_{0}\right)^{4}} 5.90 \times 10^{92} \mathrm{~g} \cdot \mathrm{cm}^{-1} \cdot \mathrm{sec}^{-2}$ $=4.95 \times 10^{-9} \mathrm{~g} \cdot \mathrm{cm}^{-1} \cdot \mathrm{sec}^{-2}=\varepsilon_{e}=\varepsilon_{v}$.

Our closed universe is now so large it is almost flat. Therefore, total energy density $\varepsilon=\varepsilon_{r}\left(\frac{R_{0}}{R}\right)^{4}+\varepsilon_{m}\left(\frac{R_{0}}{R}\right)^{3}+\varepsilon_{v}$ today is close to critical energy density $\varepsilon_{c}$ associated with a flat universe. Since $\varepsilon_{r}=4.4 \times 10^{-34} \mathrm{~g} / \mathrm{cm}^{3} c^{2}$, $\varepsilon_{m} \approx \varepsilon_{v} \approx 10^{-30} \mathrm{~g} / \mathrm{cm}^{3} c^{2}$ and $R_{0} \approx 10^{28} \mathrm{~cm}$, radiation energy density is negligible today. So, total energy density today $\varepsilon_{m}\left(\frac{R_{0}}{R}\right)^{3}+\varepsilon_{v} \approx \varepsilon_{c}$. Critical density $\rho_{c}$ relates to Hubble constant $H_{0}$ by $\rho_{c}=\frac{3 H_{0}^{2}}{8 \pi G}$. If $H_{0}=65 \mathrm{~km} \cdot \mathrm{sec}^{-2} \cdot \mathrm{Mpc}^{-2}$, $\rho_{c}=7.94 \times 10^{-30} \mathrm{~g} / \mathrm{cm}^{3}$ and critical energy density $\varepsilon_{c}=\rho_{c} c^{2}=7.15 \times 10^{-9} \mathrm{~g} \cdot \mathrm{cm}^{2} \cdot \mathrm{sec}^{-2} \cdot \mathrm{cm}^{-3}$. Thus, the dark energy (vacuum energy) fraction $\Omega_{\Lambda}=\frac{\varepsilon_{v}}{\varepsilon_{c}} \approx 0.7$. 